\title{
Article
}

\section{The Critical Capture Velocity of Coal Ash Particles Oblique Impact on a Stainless Steel Surface}

\author{
Jun Xie ${ }^{1, * \mathbb{C}}$, Haodong Ma ${ }^{1} \mathbb{D}$, Chenxi $\mathrm{Li}^{1}{ }^{1}$, Shaobai $\mathrm{Li}^{1, *}$, Zhengren $\mathrm{Zhu}^{1}$ and Zheng $\mathrm{Fu}^{2}$ \\ 1 School of Energy and Environment, Shenyang Aerospace University, Shenyang 110136, China; \\ mahaodong0216@163.com (H.M.); lichenxi961209@163.com (C.L.); zhuzhengren@yeah.net (Z.Z.) \\ 2 SPIC Northeast Electric Power Development Company Limited, Shenyang 110181, China; fz1618@aliyun.com \\ * Correspondence: xiejun@sau.edu.cn (J.X.); lishaobai1982@163.com (S.L.)
}

Citation: Xie, J.; Ma, H.; Li, C.; Li, S.; Zhu, Z.; Fu, Z. The Critical Capture Velocity of Coal Ash Particles Oblique Impact on a Stainless Steel Surface. Energies 2021, 14, 5231. https:// doi.org/10.3390/en14175231

Academic Editor: Rajender Gupta

Received: 20 July 2021

Accepted: 22 August 2021

Published: 24 August 2021

Publisher's Note: MDPI stays neutral with regard to jurisdictional claims in published maps and institutional affiliations.

Copyright: (C) 2021 by the authors. Licensee MDPI, Basel, Switzerland. This article is an open access article distributed under the terms and conditions of the Creative Commons Attribution (CC BY) license (https:/ / creativecommons.org/licenses/by/ $4.0 /)$.

\begin{abstract}
In this paper, the rebound characteristics of coal ash particles impacting on a stainless steel surface are studied experimentally with the background of ash deposition on the heating surface of the boiler. The impact processes of coal ash particles with different incident angles were recorded by high-speed digital camera technology. The evolution of the normal restitution coefficient with incident normal velocity was obtained. Three different static contact theories are used to establish the equations of motion to predict the critical capture velocity of particles. The results show that the normal restitution coefficient first increases and then decreases with the increase of incident normal velocity. The critical capture velocity of particles under the three models was predicted. It is found that the prediction results of the Brach and Dunn (BD) model for the critical capture velocity are close to the experimental results. Taking the particle of size $23 \mu \mathrm{m}$ as an example, the maximum critical capture velocity predicted by BD model is $1.0611 \mathrm{~m} / \mathrm{s}$ at $0^{\circ}$ incident angle. The minimum value is $0.7940 \mathrm{~m} / \mathrm{s}$ when the incident angle is $45^{\circ}$. The critical capture velocity of particles decreases with the increase of incident angle and with the increase of particle diameter.
\end{abstract}

Keywords: particle impact; fly ash; incident angle; normal restitution coefficient; critical capture velocity

\section{Introduction}

Particle deposition is a basic physical phenomenon, which exists widely in our production and life. In the field of energy and environment, various particle diameters of fly ash will be produced in the process of coal-fired power generation, which is easy to deposit on the heating surface, resulting in slagging, ash deposition, corrosion, and other problems leading to the reduction of boiler efficiency and the decrease of the lifespan of the heat exchange tube. Nowadays, the capture and removal of particulate matter have become an urgent scientific problem to be solved. Therefore, it is of great significance to study the dynamic mechanism of coal ash particles impacting on a smooth surface in microscale for effectively controlling the ash deposition on the surface of the heat exchanger.

To study the dynamic contact mechanism between particles and walls, some scholars at home and abroad have carried out experimental studies on the impact process between particles and walls at room temperature. Dahneke [1-3] experimentally studied the impact process of an uncharged polystyrene latex microsphere on a flat surface of different materials in a vacuum environment. In the experiment, the incident velocity and rebound velocity of particles were directly measured, the influence of adhesion on the process of particles impacting the surface of the plate was mainly considered, and the critical capture velocity was defined. When the incident velocity of particles was greater than the critical capture velocity, the particles would rebound after the collision. When the incident velocity of particles is less than the critical capture velocity, the particles are captured by the plate after a collision. Rogers and Reed [4] experimentally measured the collision process of glass, copper, and stainless steel in different combinations and obtained the corresponding critical capture velocity. Dunn et al. [5] carried out normal collision experiments to study 
the influence of the normal restitution coefficient of particles on the collision process. The results showed that for a smooth surface, the normal restitution coefficient of particles increased with the increase of incident velocity. However, due to the high incidence velocity of particles in the experiment, the phenomenon of adhesion did not occur. Li et al. [6] experimentally studied the impact process of stainless steel particles on silicon flat surfaces. It was found that the adhesion effect of the stainless steel particles with small particle diameter was more obvious than that of the stainless steel particles with large particle diameter. The adhesion effect and energy dissipation resulted in a significant decrease in the restitution coefficient. The change rule of critical capture velocity with particle diameter is also analyzed. Zhang et al. [7] used a high-speed camera to photograph the process of micron-sized graphite particles impacting with the wall surface, and discussed the critical sticking velocity and restitution coefficient under different particle diameter distributions. The results show that the critical sticking velocity decreases with the increase of particle diameter, and the restitution coefficient first increases and then decreases with the increase of incident angle. With the increase of particle diameter, the peak value of restitution coefficient decreases. Kim and Dunn [8] used high-speed digital camera technology to study the collision process between silver-plated glass particles and silica surfaces and obtained the relationship between normal restitution coefficient and incident velocity. Sommerfeld et al. [9] studied the impact of 60-micron quartz particles on stainless steel and Ti6Al4V targets. The effects of impact velocity on the rebound characteristics were analyzed. The results show that the absolute restitution coefficient decreases with the increase of impact velocity. Moreover, the particle has the phenomenon of fragment, and the fragmentation increases with the increase of the particle impact velocity and impact angle. Tiamiyu et al. [10] studied the rebound characteristics of copper particles impacting with copper surface at ultra-high velocity, and they showed that when the incident velocity was between $100 \mathrm{~m} / \mathrm{s}$ and $590 \mathrm{~m} / \mathrm{s}$, the particle recovery coefficient decreased with the increase of incident velocity. Wall et al. [11] used Laser Doppler measurement technology to measure the incident velocity and rebound velocity of luciferin ammonium particles in the process of collision with different materials and obtained the critical capture velocity of particles under different working conditions. Dong et al. [12] studied the rebound behavior of fly ash particles impact a plane surface in the normal direction, and they obtained the critical capture velocity of particles with different particle diameters. Li et al. [13] studied the critical capture velocity of silica particle impact on the surface at different temperatures and found that the surface temperature would affect the critical capture velocity of particles. Li et al. [14] analyzed the restitution coefficient of various fly ash particles with diameters of 7 microns impacting the plate surface under different humidity conditions. For all coal ash particles under all working conditions, the normal restitution coefficient showed a trend of first increasing and then decreasing with the increase of incident velocity.

Konstandopoulos [15] studied and analyzed the oblique collision process of spherical particles impacting the surface and calculated the rebound process or capture process of particles after the oblique collision through the particle motion equation containing the contact mechanism. Aylor and Ferrandino [16] studied the bouncing process of arragweed pollen and lycophysidia spores on the cylinder. The results show that both tangential incident velocity and normal incident velocity have effects on the rebound characteristics of particles. The restitution coefficient will change with the change of the impact position on the surface of the cylinder.

Gibson et al. [17] experimentally studied the process of particle impact on the wall and measured the normal restitution coefficients and tangential restitution coefficients of the particle. When the particles impact the wall, the motion of the particles is decomposed into two directions: normal and tangential. This process is considered to be the main reason for the rotation of the particles, which leads to the reduction of the normal restitution coefficient. Under this background, it can be concluded that the sphericity of particles has a significant effect on the diameter of the restitution coefficient. In addition, the material properties of the impact platform also have a certain influence on the rebound angle and 
restitution coefficient. The expressions of normal restitution coefficient and tangential restitution coefficient are given in this paper. Kuuluvainen et al. [18] studied the effects of materials and incident angles on the critical capture velocity of luciferin ammonium particles after impacting on different surfaces and defined the concept of inclination. The results showed that the critical capture velocity of luciferin ammonium particles was largely affected by inclination.

Sondergaard et al. [19] studied the rebound characteristics of glass particles and stainless steel particles with different particle diameters when they impact the aluminum surface at different velocities and angles. The motion trajectory of the particles was filmed by a camera. In the later period, the incident velocity and rebound velocity of the particles were obtained through data analysis. Xie et al. $[19,20]$ studied the impact process of silicon dioxide particles on stainless steel at different incident velocity and angle, and they obtained the critical capture velocity under different working conditions. From the perspective of energy loss, they concluded that the critical capture velocity of particles at high incident angle was smaller. Brach et al. [21] experimentally studied the process of glass particle impact aluminum surface at different incident angles and found that the restitution coefficient was higher when the particle impact aluminum surface at a smaller incident angle. Beek et al. [22] selected glass particles to impact with glass plates. The effects of incident velocity and incident angle on the normal restitution coefficient were studied experimentally. The results show that the normal restitution coefficient decreases with the increase of incident angle.

Dunn et al. [5] carried out an experimental study on micron-sized particles obliquely impacting the flat surface. For smooth surfaces, the tangential effect of particles impacting the flat surface was enhanced by the spin of particles and the surface roughness of the flat surface, leading to the increase of the restitution coefficient of particles with the increase of the initial normal velocity at a small incident angle. Li et al. [23] used the same experimental system to carry out an experimental study on the impact of stainless steel particles on a smooth silicon surface, and 11 velocity conditions were designed. It is found that when the incident velocity of particles is close to the critical capture velocity, the adhesion effect of small particle diameter is more obvious than that of large particle diameter. The critical capture velocity of particles is also different in the case of normal collisions and oblique collisions.

Many scholars have carried out experimental research on the impact of micro-scale particles on the rigid wall surface, but the incident particle materials used are different from the actual situation in coal-fired power plants, which cannot well reflect the actual situation. In this paper, coal ash particles from coal-fired power plants were selected to record the collision process between coal ash particles and stainless steel surface at different incident angles by using high-speed digital camera technology. The influence of incident normal velocity on the normal restitution coefficient is analyzed, and the factors affecting the normal restitution coefficient at different incident normal velocities are discussed. In addition, based on Hertz theory, Derjaguin, Muller, and Toporov (DMT) theory, and BD theory [24-26], the motion equation is established to predict the critical damping coefficient and the critical capture velocity of coal ash particles impacting on the stainless steel surface. Finally, the influence of particle diameter and the incident angle on the critical capture velocity is analyzed. This provides a basic condition for reasonably predicting the adhesion efficiency of coal ash particles in coal-fired power plants and predicting the ash deposition process, and it also provides a theoretical basis for effectively controlling the ash deposition in boilers.

\section{Experimental System and Methods}

\subsection{Experimental System}

An experimental device for coal ash particles impacting the stainless steel surface was developed. Figure 1 shows the schematic diagram of the experimental system in which coal ash particles impact a flat plate surface. The system consists of a nitrogen gas 
source, a particle syringe, a light source, a high-speed camera, and a stainless steel impact plate. The particles syringe is prepared for a small number of fly ash particles after drying treatment in advance of the process of the experiment, and then the nitrogen cylinder pressure reducer and the manual control valve were opened. Then, open-air ventilation with nitrogen purging was carried out on the surface, with a small waiting time to allow the nitrogen to flow smoothly. We regulated the flow of nitrogen gas and the mass flow controller and monitored the nitrogen flow; its measurement precision is $\pm 1 \%$.

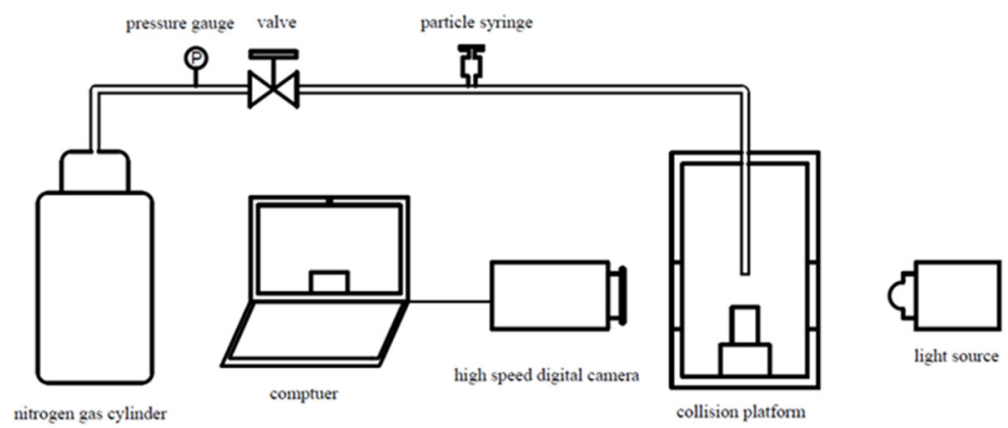

Figure 1. Schematic diagram of experimental system in which coal ash particles impact with flat plate surface.

The nitrogen flow rate in this experiment was controlled from $0.35 \mathrm{~L} / \mathrm{min}$ to $0.7 \mathrm{~L} / \mathrm{min}$, and the flow interval was changed by $0.05 \mathrm{~L} / \mathrm{min}$. When the nitrogen flow reaches the predetermined flow value, we slowly pushed the particle syringe to transport the coal ash particles into the pipeline, and the coal ash particles are transported to the top of the impact cylinder by nitrogen to collide with the stainless steel surface at the set angle below to ensure that the flow direction of nitrogen airflow is perpendicular to the horizontal plane at this time. The impact platform used in this experiment is made of a SUS316 stainless steel cylinder with a diameter of $2 \mathrm{~mm}$ through the milling process. In the process, the roughness of the surface is guaranteed to be less than $0.2 \mu \mathrm{m}$, so it can be regarded as a smooth surface. The inclination angle $\alpha$ of impacting the platform surface was selected as $0^{\circ}, 30^{\circ}$, and $45^{\circ}$ (the inclination angle $\alpha$ was defined as the included angle between the inclined plane and the horizontal plane). Figure 2 shows the picture taken when the inclination angle was $45^{\circ}$. The nitrogen gas source is provided by Dalian Yongfeng Gas Cobtd, the volume of the cylinder is $40 \mathrm{~L}$. The volume of the particle syringe is $1 \mathrm{~mL}$. The cold light source of the XD-300 xenon lamp was selected as the cold light source for the shooting. (Cold light source power $250 \mathrm{~W}$, illumination 5,800,000 lx, the brightness can be adjusted.) The collision process between coal ash particles and the stainless steel surface was filmed in real-time with a high-speed digital camera. The video captured was stored in the computer for subsequent data analysis. The high-speed digital camera used in the experiment is produced by a VRI company in the United States, the model is Phantom V12.1, equipped with a VS-M9010 lens. The specific technical indicators of the high-speed digital camera are described in Table 1.

Table 1. Specific technical indexes of high-speed digital camera.

\begin{tabular}{lll}
\hline Serial Number & Indicators & Parameter \\
\hline 1 & Resolution ratio & $256 \times 256$ \\
2 & Shooting rate & $66,037 \mathrm{fps}$ \\
3 & Exposure time & $14.705 \mu \mathrm{s}$ \\
4 & Embedded memory capacity & $8 \mathrm{G}$ \\
\hline
\end{tabular}




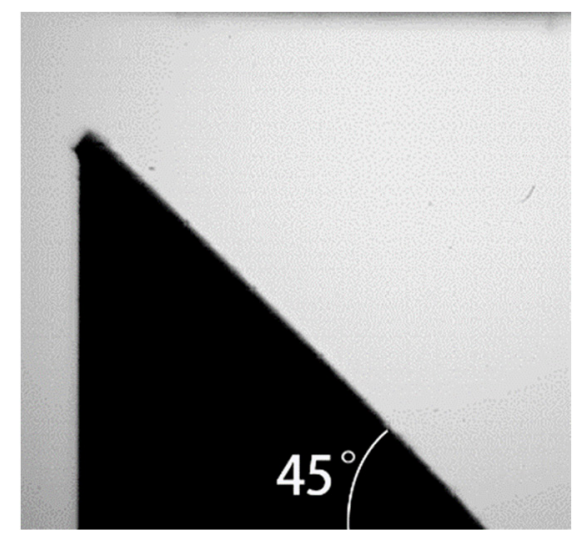

Figure 2. Impact platform with inclination angle of $45^{\circ}$.

The coal ash particles in the experiment came from the thermal power plant and were sampled in front of the dust collector of the boiler and screened by a vibrating screen machine. In this experiment, the coal ash particle mesh number is less than 325 mesh, particle density is $2100 \mathrm{~kg} / \mathrm{m}^{3}$, Poisson's ratio is 0.21 , and elastic modulus is $134 \mathrm{GPa}$. Figure 3 shows the whole process of coal ash particles bouncing back after the incident from the direction perpendicular to the stainless steel surface and impacting the target surface.

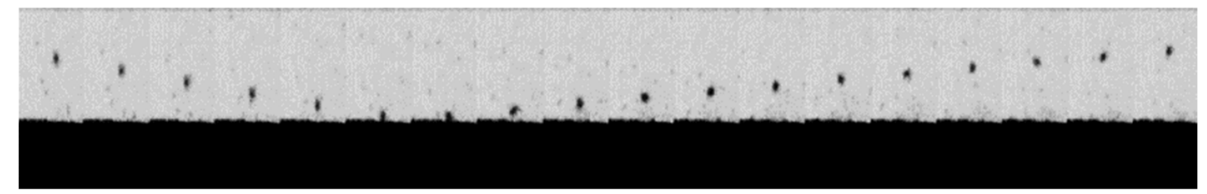

Figure 3. Process of coal ash particles impacting the stainless steel surface in normal direction.

\subsection{Parameter Measurement}

The experiment needs to measure the incident velocity $V_{i}$, the rebound velocity $V_{r}$ of the particles, the incident angle $\theta_{i}$ and the rebound angle $\theta_{r}$, and the particle diameter $d_{p}$. During data processing, coal ash particles impacting on the stainless steel surface in a direction perpendicular to the horizontal plane were manually selected.

To obtain the incident velocity $V_{i}$ and the rebound velocity $V_{r}$, three images before and after the collision were selected for calculation. Using measure incident velocity as an example, the position of pixels of coal ash particles on three images is measured by Adobe Photoshop software, and the pixel displacement $\Delta s$ of coal ash particles on every two images is calculated and the average value is taken, and then divided by the interval time $\Delta t$ between two images so that the relative incidence velocity of coal ash particles can be calculated. Note that the displacement calculated by this method is the displacement in the image, which needs to be scaled to get the actual displacement and then to calculate the actual velocity. When processing the data, high-speed digital camera video postprocessing software is used to preprocess the video shot. The impact platform with a diameter of $2 \mathrm{~mm}$ is selected as the reference, and the pixel value represented by $2 \mathrm{~mm}$ is calculated as $L_{\text {pixel }}$. Then, the expression of the actual velocity can be obtained as follows:

$$
V=\frac{\Delta s_{1}+\Delta s_{2}}{L_{\text {pixel }} \Delta t}
$$

The incident angle $\theta_{i}$ is defined as the acute angle between the incident velocity of the particle and the normal direction of the plate surface. The rebound angle $\theta_{r}$ is defined as the acute angle between the rebound velocity of the particle when it disentangles and the normal release of the plate surface. The details are shown in Figure 4. The angle measurement can be calculated by the intersection of the fitting curve of the pixel 
coordinates of coal ash particles in Excel and the fitting curve of the plate normal on the three images.

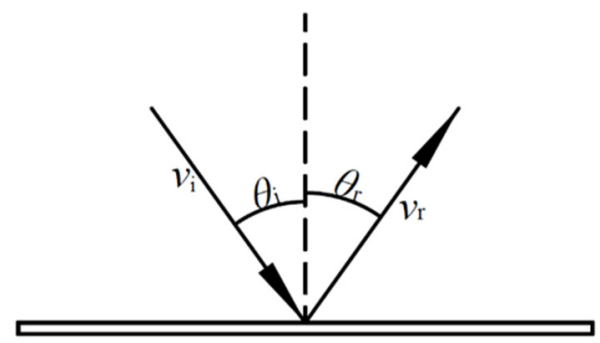

Figure 4. Definition of velocity and angle of coal ash particles impact the stainless steel surface.

The measurement of particle diameter $d_{p}$ is determined by the method of pixel value calibration. Adobe Photoshop is used to measure the pixel area value $s_{\text {pixel }}$ of the particle in the image, and the particle diameter can be obtained after proportional conversion. The calculation formula is as follows:

$$
\begin{gathered}
d_{\text {pixel }}=\sqrt{\frac{4 s_{\text {pixel }}}{\pi}} \\
d_{p}=\frac{2 d_{\text {pixel }}}{L_{\text {pixel }}}
\end{gathered}
$$

The relationship between the normal restitution coefficient and the incident normal velocity is one of the main indicators to describe particle collision. Then, the expression of the normal restitution coefficient $e_{n}$ is as follows:

$$
e_{n}=\frac{V_{r n}}{V_{i n}}
$$

where $V_{\text {in }}$ is the incident normal velocity and $V_{r n}$ is the rebound normal velocity.

\subsection{Numerical Calculation Method}

There are two outcomes when coal ash particles collide with the stainless steel surface: adhesion or rebound. To describe the collision mechanism between the particle and plate surface, according to the interaction force between particle and plate in quasi-static contact, the dynamic contact model and the motion equation of particle are established. Figure 5 is a schematic diagram of the contact process between two particles. The central normal distance of the intersecting part of two particles is defined as the contact potential, which can be expressed as

$$
x=r_{1}+r_{2}-d
$$

where $r_{1}, r_{2}$ are the radii of the first particle and second particle, and $d$ is the center distance of the two particles.

Hertz first established the static contact model in the normal collision process of two elastic particles. The Hertz model considers that the deformation of particles only occurs within the contact region, and it only considers the influence of repulsive force on the contact process of two particles but does not consider the influence of attractive force on the contact process of two particles. The total force $F^{H}$ in the contact area can be deduced from the Hertz model as follows [24]:

$$
F^{H}=\frac{4}{3} E^{*} R^{* 1 / 2} x^{3 / 2}
$$


where $E^{*}$ is the equivalent elastic modulus of two particles and $R^{*}$ is the equivalent radius of two particles. Both can be calculated by the following formula:

$$
\begin{gathered}
\frac{1}{E^{*}}=\frac{1-\gamma_{1}}{E_{1}}+\frac{1-\gamma_{2}}{E_{2}} \\
\frac{1}{R^{*}}=\frac{1}{r_{1}}+\frac{1}{r_{2}}
\end{gathered}
$$

where $E_{1}, E_{2}$ are the elastic modulus of first particle and second particle, $\gamma_{1}, \gamma_{2}$ are the Poisson's ratio of first particle and second particle, and $r_{1}, r_{2}$ are the radius of first particle and second particle.

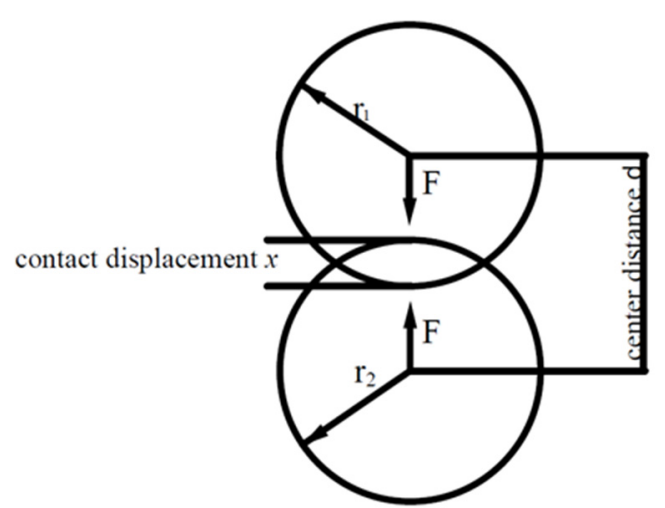

Figure 5. The contact process between two particles.

The Hertz model does not take into account the attractive force between two particles, but because the surface of particles has surface energy, there is a tendency for particles to adhere, so the attractive force between particles exists. Derjaguin, Muller, and Toporov proposed an adhesive theory, known as the DMT theory. The DMT model considers not only the influence of repulsive force on the collision process, but also the influence of attractive force on the collision process. The DMT model deals with the repulsive force based on Hertz theory. For the treatment of attractive force, the DMT model believes that attractive force acts directly outside the contact region of two particles. According to the DMT model, the total force $F^{D M T}$ in the contact area is equal to the combined force of the gravitational forces $F_{a}^{D M T}$ and repulsive forces $F_{r}^{D M T}$. The attractive forces $F_{a}^{D M T}$ and the repulsive forces $F_{r}^{D M T}$ can be calculated by the following formula [25]:

$$
\begin{gathered}
F_{a}^{D M T}=-2 \pi \omega R^{*} \\
F_{r}^{D M T}=\frac{4}{3} E^{*} R^{* 1 / 2} x^{3 / 2}
\end{gathered}
$$

where $\omega$ is the adhesion work per unit area, and it can be calculated from the surface energy of two materials in contact with particles, and the expression is as follows:

$$
\omega=\sqrt{\kappa_{1} \kappa_{2}}
$$

where $\kappa_{1}, \kappa_{2}$ are the surface energies of first particle and second particle.

The total force $F^{D M T}$ in the contact area calculated by the DMT model is as follows:

$$
F^{D M T}=F_{a}^{D M T}+F_{r}^{D M T}
$$

Brach and Dunn proposed the BD model. The BD model is similar to the DMT model, considering the repulsion force based on Hertz theory, but the BD model asserts that an attractive force acts within the contact region of two particles. Correspondingly, from the $\mathrm{BD}$ model, it can be concluded that the total force $F^{B D}$ in the contact area is equal to the 
combined force of gravitational forces $F_{a}^{B D}$ and repulsive forces $F_{r}^{B D}$. The attractive forces $F_{a}^{B D}$ and the repulsive forces $F_{r}^{B D}$ can be calculated by the following formula [26]:

$$
\begin{gathered}
F_{a}^{B D}=-2 \pi R^{* 1 / 2} x^{1 / 2}\left(\frac{6 \omega^{2} R^{*} E^{*}}{\pi}\right)^{1 / 3} \\
F_{r}^{B D}=\frac{4}{3} E^{*} R^{* 1 / 2} x^{3 / 2}
\end{gathered}
$$

The total force $F^{B D}$ in the contact area calculated by the $\mathrm{BD}$ model can be expressed as

$$
F^{B D}=F_{a}^{B D}+F_{r}^{B D}
$$

Based on the theoretical framework of the Hertz model, DMT model, and BD model, and considering the influence of energy dissipation, the motion equation of the collision process of two particles is established as follows:

$$
m^{*} \frac{d^{2} x}{d t^{2}}+\eta \frac{d x}{d t}+F=0
$$

where $\eta$ is the damping coefficient; $F$ is the resultant force substituted into the physical model; $t$ is the time interval, it starts with the moment when the two particles just start to contact each other and do not deform and ends with the moment when the two particles are just divided; and $m^{*}$ is the effective mass of two particles, which can be calculated by the following formula:

$$
\frac{1}{m^{*}}=\frac{1}{m_{1}}+\frac{1}{m_{2}}
$$

where $m_{1}, m_{2}$ are the masses of first particle and second particle.

All the models described above are based on the collision process between two particles. For the collision process between coal ash particles and the stainless steel surface described in this paper, the diameter of coal ash particles selected is much smaller than that of the stainless steel surface, and the quality of coal ash particles selected is also much smaller than that of the stainless steel surface. Therefore, the stainless steel surface can be regarded as an infinite radius and an infinite mass of particles. In this case, the effective radius is equal to the radius of coal ash particles, and the effective mass is equal to the mass of coal ash particles: $R^{*}=r_{1}, m^{*}=m_{1}$.

The above equation of motion is discretized according to time term, and the display format of the equation of motion is obtained as follows:

$$
\frac{x_{i}-2 x_{i+1}+x_{i+2}}{\Delta t^{2}}+\frac{\eta}{m_{1}} \frac{x_{i+2}-x_{i}}{2 \Delta t}+\frac{F_{i+1}}{m_{1}}=0
$$

where $\Delta t$ is the interval time.

If the displacement at one moment $x_{i}$ and the displacement at the next moment $x_{i+1}$ are known, the displacement at the next two moments $x_{i+2}$ can be calculated. The displacement at the initial moment is defined as $x_{0}$, the velocity $v_{0}$ is the incident normal velocity, and the time interval is taken $\Delta \mathrm{t}=1 \mathrm{~ns}$. As the time interval selected is small enough and the collision deformation is very small at the next moment, the motion of particles from the initial moment to the next moment can be considered to be a uniform linear motion. Therefore, the displacement of the process at this stage can be obtained by the formula $x_{1}=v_{0} \Delta t$, and the velocity at each moment and the displacement of the process at each stage can be calculated. The condition of collision termination is set as when the displacement at any time except the initial time is infinitely close to 0 . In the calculation process, the damping coefficient is assumed to be a certain value and the initial velocity $v_{0}$ is input to calculate the velocity $v_{n}$ at the termination moment of the collision. By calculating the ratio of the terminal velocity $v_{n}$ to the initial velocity $v_{0}$, the normal restitution coefficient can be expressed as $e=\frac{v_{n}}{v_{0}}$. By comparing it with the normal 
restitution coefficient $e_{n}$ obtained from the experiment, until the error of the two results is less than $0.1 \%$, it can be considered to be the same, and then the damping coefficient can be output. If there is a big difference between the two results, the damping coefficient needs to be re-assumed until the error reaches the set value, and the damping coefficient can be output. The flow chart of numerical calculation is shown in Figure 6, and the detailed expressions for calculating the critical damping coefficient under the Hertz model, DMT model, and BD model are as follows:

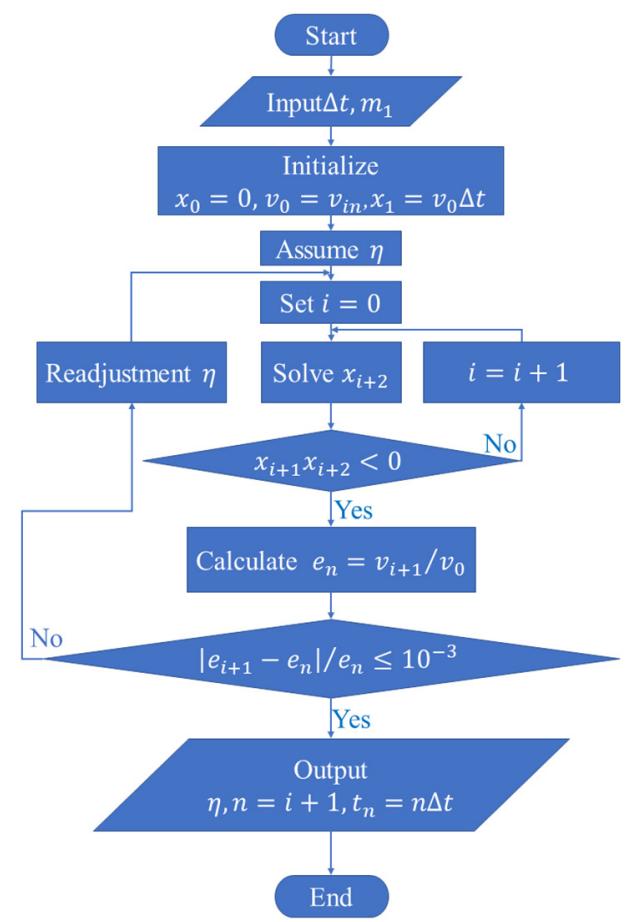

Figure 6. Flow chart for solving the damping coefficient.

For the Hertz model:

$$
\frac{x_{i}-2 x_{i+1}+x_{i+2}}{\Delta t^{2}}+\frac{\eta}{m_{1}} \frac{x_{i+2}-x_{i}}{2 \Delta t}+\frac{4}{3} \frac{E^{*} r_{1}^{1 / 2}}{m_{1}} x_{i+1}^{3 / 2}=0
$$

For the DMT model:

$$
\frac{x_{i}-2 x_{i+1}+x_{i+2}}{\Delta t^{2}}+\frac{\eta}{m_{1}} \frac{x_{i+2}-x_{i}}{2 \Delta t}+\frac{4}{3} \frac{E^{*} r_{1}^{1 / 2}}{m_{1}} x_{i+1}^{3 / 2}-\frac{2 \pi \omega r_{1}}{m_{1}}=0
$$

For the BD model:

$$
\frac{x_{i}-2 x_{i+1}+x_{i+2}}{\Delta t^{2}}+\frac{\eta}{m_{1}} \frac{x_{i+2}-x_{i}}{2 \Delta t}+\frac{4}{3} \frac{E^{*} r_{1}^{1 / 2}}{m_{1}} x_{i+1}^{3 / 2}-\frac{2 \pi^{2 / 3} r_{1}\left(6 \omega^{2} R^{*} E^{*}\right)^{1 / 3}}{m_{1}} x_{i+1}^{1 / 2}=0
$$

\section{Results and Discussion}

\subsection{Normal Restitution Coefficient}

Figure 7 shows the variation between the normal restitution coefficient and the incident normal velocity. The impact of the incident angle $0^{\circ}$ and $30^{\circ}$ for $23 \mu \mathrm{m}, 28 \mu \mathrm{m}$, and $32 \mu \mathrm{m}$ of coal ash particles were selected, while the impact of the incident angle $45^{\circ}$ is for $8 \mu \mathrm{m}$, $23 \mu \mathrm{m}$, and $28 \mu \mathrm{m}$. According to the slope change of the curve, it can be divided into ascending region, transition region, and descending region. In the ascending region, the curve has a large slope, and with the increase of the incident normal velocity, the normal restitution coefficient increases, but the slope of the curve decreases. In the transition region, with the increase of the incident normal velocity, the normal restitution coefficient 
first increases and then decreases, and the peak value of the normal restitution coefficient appears in this region. Besides, the positive and negative values of the curve slope change in the transition region. In the curve descending region, with the increase of the incident normal velocity, the normal restitution coefficient decreases, and the absolute value of the curve slope increases. The relationship between the normal restitution coefficient and the incident normal velocity is consistent with the conclusion of Zhang et al. and Li et al. [7,14], but the peak value of the restitution coefficient is different because of the difference between the particle and the plate surface materials.

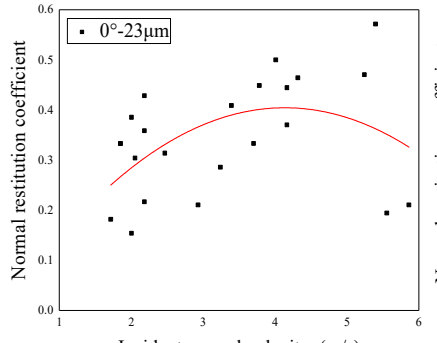

Incident normal velocity $(\mathrm{m} / \mathrm{s})$

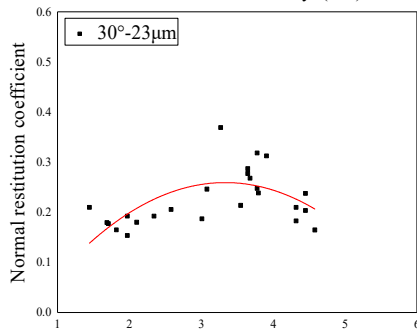

Incident normal velocity $(\mathrm{m} / \mathrm{s})$

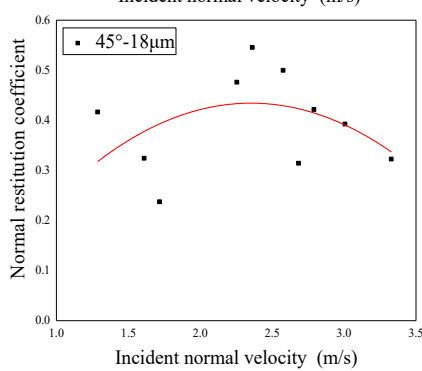

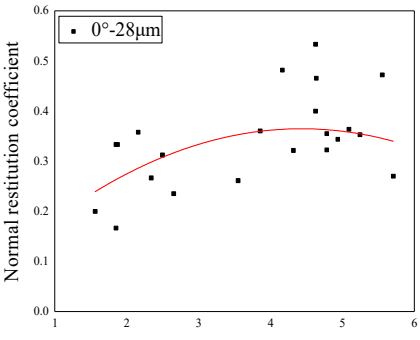

Incident normal velocity $(\mathrm{m} / \mathrm{s})$

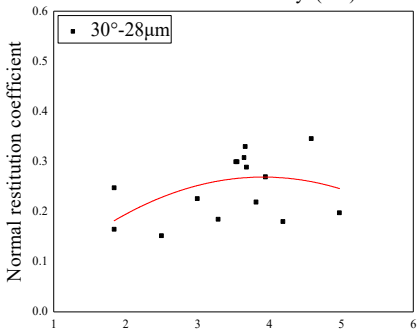

Incident normal velocity $(\mathrm{m} / \mathrm{s})$

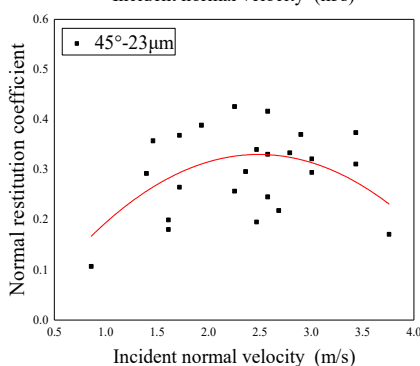

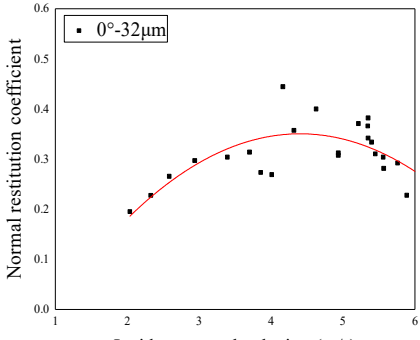

Incident normal velocity $(\mathrm{m} / \mathrm{s})$

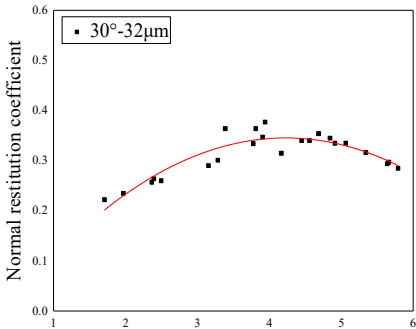

Incident normal velocity $(\mathrm{m} / \mathrm{s})$

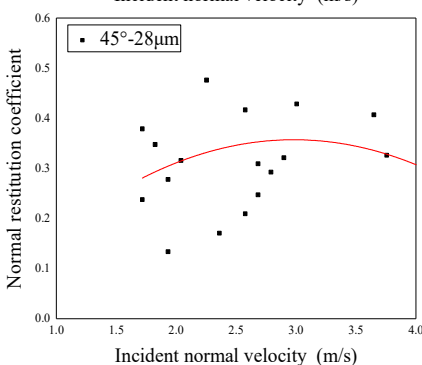

Figure 7. The ash particles with different particle sizes impact on the $e_{n}-V_{\text {in }}$ curve of the stainless steel surface at different incident angles.

Thomton and Ning [27] gave the exact expressions of the restitution coefficients for different velocity intervals. If $v_{p}^{*} \leq V_{i n}<v_{y}, e_{n}=\left[1-\left(\frac{v_{p}^{*}}{V_{i n}}\right)^{2}\right]^{0.5}$, and if $v_{y} \leq V_{i n}$, $e_{n}=\frac{6 \sqrt{3}}{5}\left[1-\frac{1}{6}\left(\frac{v_{y}}{V_{i n}}\right)^{2}\right] \times\left[\frac{\frac{v_{y}}{V_{i n}}}{\frac{v_{y}}{V_{\text {in }}}+2 \sqrt{\frac{6}{5}-\frac{1}{5}\left(\frac{v_{y}}{V_{i n}}\right)^{2}}}\right]^{0.5}-\left(\frac{v_{p}^{*}}{V_{i n}}\right)^{2}$. Based on the analysis of Figure 5, when the incident normal velocity between the critical capture velocity and plastic yield velocity, the restitution coefficients increase with the increase of the incident normal velocity. When the incident normal velocity approach the critical capture velocity, $\partial e_{n} / \partial V_{\text {in }}$ very large, the slope of the curve is large. With the further increase of the incident normal velocity, $\partial e_{n} / \partial V_{i n}$ is reduced and the slope of the curve decreases. At this point, the ascending region of the curve follows a similar trend. When the incident normal velocity is greater than the plastic yield velocity, the restitution coefficients decrease with increasing incident normal velocity. At this point, the descending region of the curve follows a similar trend. The plastic yield velocity is in the transition region of the curve. 


\subsection{Prediction of Critical Capture Velocity by the Three Models}

\subsubsection{Critical Damping Coefficient}

To reduce the calculation error, the incident normal velocity and the normal restitution coefficient of particles are not selected directly in the basic data of the calculation of damping coefficient, but the incident normal coefficient and the normal restitution coefficient are optimized by combining the fitting curve. In the calculation, 15 groups of incident normal velocities were preselected, and the corresponding normal restitution coefficient was calculated by fitting the polynomial equation of the curve, and then the basic data of this group were used for the following calculation. The physical parameters of the coal ash particles and stainless steel surface required in the calculation are shown in Table 2. The material property parameters used in the calculation were derived from the coal ash particles and stainless steel plate used in the experiment. The density of coal ash particles is measured by an automatic true density analyzer. The surface energy of the particles and the surface energy of the stainless steel plate were analyzed by measuring the contact angle with the two-liquid method. The measurement of elastic modulus of coal ash particles refers to the X-ray diffraction experiment of Matsunaga et al. [28], the crystal phase and mass percentage of coal ash are analyzed, and the elastic modulus is calculated by using the parallel model of mixture. The Poisson's ratio of coal ash particles was measured by electrical method. The elastic modulus and Poisson's ratio of stainless steel plate refer to Ledbetter's [29] conclusion.

Table 2. Basic physical properties of coal ash particles and flat surface.

\begin{tabular}{cccc}
\hline Serial Number & $\begin{array}{c}\text { Basic Physical } \\
\text { Property Parameters }\end{array}$ & Unit & $\begin{array}{c}\text { Physical Parameter } \\
\text { Values }\end{array}$ \\
\hline 1 & Particle density & $\mathrm{kg} / \mathrm{m}^{3}$ & 2100 \\
2 & Modulus of elasticity of particles & $\mathrm{GPa}$ & 134 \\
3 & Poisson's ratio of particles & - & 0.21 \\
4 & Particle surface energy & $\mathrm{kJ} / \mathrm{m}^{2}$ & 89.75 \\
5 & Modulus of elasticity of plate & $\mathrm{GPa}$ & 206 \\
6 & Poisson's ratio of the plate & - & 0.29 \\
7 & Plate surface energy & $\mathrm{kJ} / \mathrm{m}^{2}$ & 247.96 \\
\hline
\end{tabular}

Figure 8 shows the relationship between the damping coefficient calculated by the three models and the normal restitution coefficient at different incidence angles. For any working condition, the damping coefficient decreases with increasing normal restitution coefficient. The purpose of calculating the relationship between damping coefficient and normal restitution coefficient is to solve the critical damping coefficient. Therefore, the incident normal velocity of 15 groups of data selected for calculation is all from the rising region of the curve in Figure 7.

According to the fitting relationship between the damping coefficient and the normal restitution coefficient in Figure 7, when the normal restitution coefficient is 0 , the corresponding critical damping coefficients of Hertz model, DMT model, and BD model at different angles can be calculated. The calculated critical damping coefficient is shown in Table 3. 

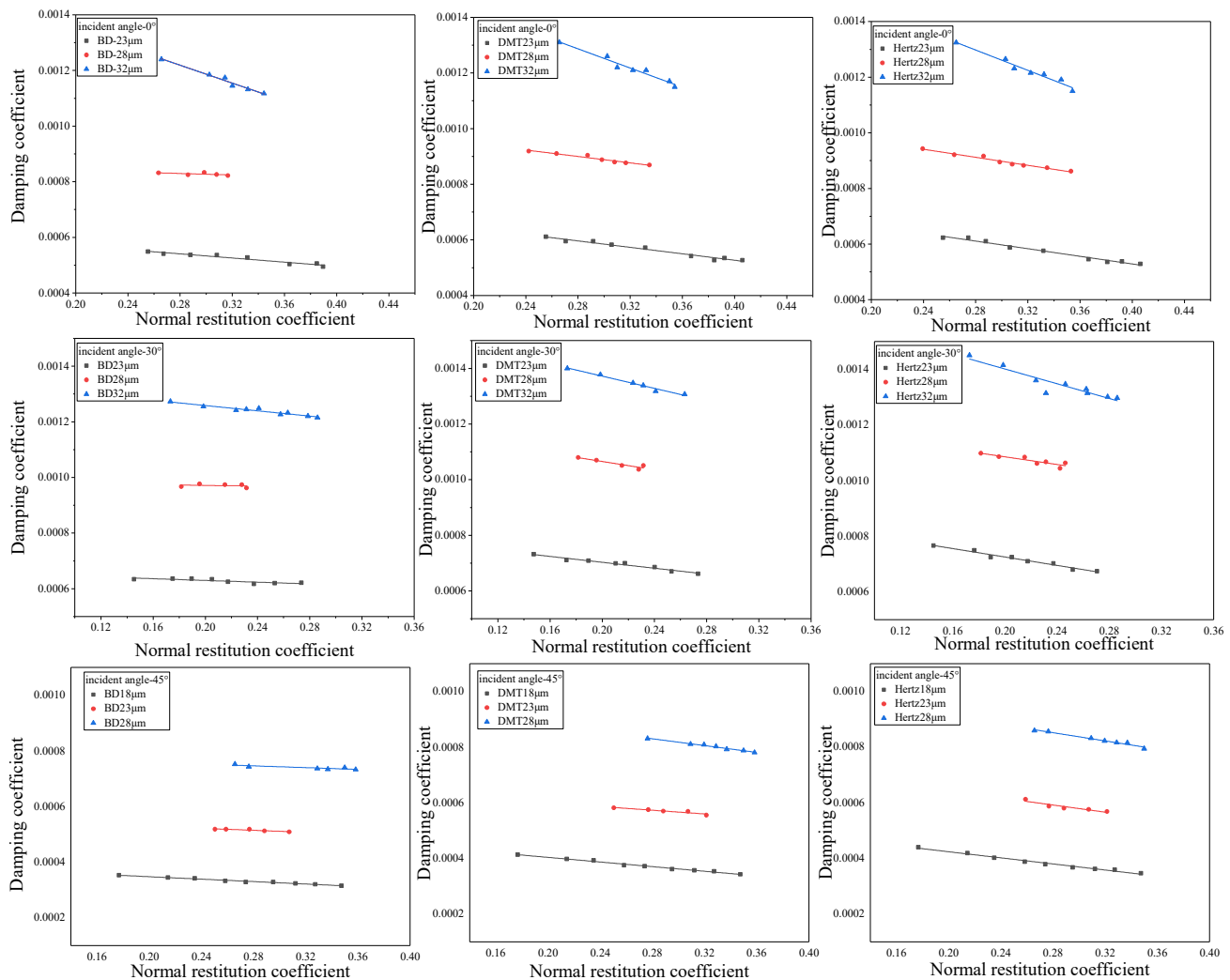

Figure 8. The relation between damping coefficient and normal restitution coefficient of Hertz model, DMT model, and BD model at different angles.

Table 3. The critical damping coefficients of Hertz model, DMT model, and BD model at different incident angles.

\begin{tabular}{llll}
\hline (a) Incident Angle $0^{\circ}$ & & & \\
\hline Diameter & Hertz Model & DMT Model & BD Model \\
\hline 23 & 0.000905 & 0.000750 & 0.000686 \\
28 & 0.001200 & 0.000947 & 0.000815 \\
32 & 0.001490 & 0.001080 & 0.000936 \\
\hline (b) Incident Angle 30 & & & \\
\hline Diameter & Hertz Model & DMT Model & BD Model \\
\hline 23 & 0.000881 & 0.000738 & 0.000624 \\
28 & 0.001170 & 0.000918 & 0.000801 \\
32 & 0.001360 & 0.001060 & 0.000836 \\
\hline (c) Incident Angle 45 & & & \\
\hline Diameter & & DMT Model & \\
\hline 18 & Hertz Model & DModel \\
23 & 0.000574 & 0.000485 & 0.000399 \\
28 & 0.000801 & 0.000667 & 0.000589 \\
\hline
\end{tabular}

\subsubsection{Critical Capture Velocity}

The critical capture velocity of particles is one of the main indicators to describe the particle impact process. When the incident normal velocity of particles is less than the critical capture velocity, the particles will adhere to the plate surface and will not rebound. When the incident normal velocity of particles is greater than the critical capture velocity, the particles will rebound. Therefore, the critical capture velocity can be used as a theoretical criterion to judge whether the particles rebound after impinging on the 
plate surface. When the initial incident velocity is less than the critical capture velocity, the normal restitution coefficient is 0 , and the particles adhere to the plate surface. When the initial incident velocity is greater than the critical capture velocity, the normal restitution coefficient increases first and then decreases with the increase of the incident normal velocity, and then the particle will rebound.

Make the critical damping coefficient of different models as a precondition and the restitution coefficient is set to 0 , then deduces the critical damping coefficient under the corresponding incident normal velocity, this incident normal velocity can be considered to be the model predicted critical capture velocity. The calculated critical capture velocity is shown in Table 4.

Table 4. The critical capture velocity of Hertz model, DMT model, and BD model at different incident angles.

\begin{tabular}{llll}
\hline (a) Incident Angle $0^{\circ}$ & & & \\
\hline Diameter & Hertz Model & DMT Model & BD Model \\
\hline 23 & 0.5725 & 0.7755 & 1.0611 \\
28 & 0.3316 & 0.4949 & 0.6437 \\
32 & 0.2589 & 0.3505 & 0.4757 \\
(b) Incident Angle 30 & & & \\
\hline Diameter & Hertz Model & DMT Model & BD Model \\
\hline 23 & 0.5020 & 0.7469 & 0.8829 \\
28 & 0.2961 & 0.4633 & 0.6151 \\
32 & 0.1651 & 0.3389 & 0.3992 \\
(c) Incident Angle 45 & & & BD Model \\
\hline Diameter & Hertz Model & DMT Model & 1.0796 \\
\hline 18 & 0.6746 & 0.9768 & 0.7940 \\
23 & 0.3133 & 0.5937 & 0.5088 \\
\hline 28 & 0.1902 & 0.3773 & \\
\hline
\end{tabular}

To verify which of the critical capture velocities of coal ash particles predicted by the three models is more consistent with the movement behavior of coal ash particles under the actual conditions of coal-fired power plants, the critical capture velocity predicted by the three models should be compared with the experimental critical capture velocity. However, it is very difficult to obtain the critical capture velocity of particles in the experiment, so the data (including incident angle, particle diameters, incident velocity, and movement behavior of particles after impacting with stainless steel surface) of coal ash particles under different working conditions in the experiment were selected, as shown in Table 5. The incident velocity of particles in the experimental data selected in Table 5 was compared with the critical capture velocity predicted by the model in Table 4 . For example, in case 3 , a $28 \mu \mathrm{m}$ coal ash particle with normal incident adhered after impacting the stainless steel surface at $0.5528 \mathrm{~m} / \mathrm{s}$, while in case 4, a $28 \mu \mathrm{m}$ coal ash particle with normal incident rebounded after impacting the stainless steel surface at $0.6508 \mathrm{~m} / \mathrm{s}$. The results show that the critical capture velocity of ash particle with $28 \mu \mathrm{m}$ normal impact on stainless steel surface is between $0.5528 \mathrm{~m} / \mathrm{s}$ and $0.6508 \mathrm{~m} / \mathrm{s}$. The critical capture velocity of coal ash particle predicted by the three models in Table 4 under this condition is $0.3316 \mathrm{~m} / \mathrm{s}$ for the Hertz model, $0.4949 \mathrm{~m} / \mathrm{s}$ for the DMT model, and $0.6437 \mathrm{~m} / \mathrm{s}$ for the BD model. This indicates that the critical capture velocity predicted by the $\mathrm{BD}$ model is close to the critical capture velocity of the actual coal ash particle collision process. The errors of the three models are, respectively, $96.26 \%$ for the Hertz model, $31.50 \%$ for the DMT model, and $1.10 \%$ for the BD model. This also shows that the critical capture velocity predicted by the $\mathrm{BD}$ model is more in line with the actual situation. For other cases, the same conclusion can be obtained by the above methods, the critical capture velocity predicted by the BD model is close to the actual situation, and the maximum error of the critical capture velocity 
predicted by the three models is $168.66 \%$ for Hertz model, $38.40 \%$ for DMT model, and $2.95 \%$ for BD model.

Table 5. The movement behavior of coal ash particles after impacting the stainless steel surface under different working conditions during the experiment.

\begin{tabular}{ccccc}
\hline Case & Incident Angle & Diameter $(\mu \mathbf{m})$ & $\begin{array}{c}\text { Incident } \\
\text { Velocity }(\mathbf{m} / \mathbf{s})\end{array}$ & Adhesion/Rebound \\
\hline 1 & $0^{\circ}$ & 23 & 0.9546 & adhesion \\
2 & $0^{\circ}$ & 23 & 1.0924 & rebound \\
3 & $0^{\circ}$ & 28 & 0.5528 & adhesion \\
4 & $0^{\circ}$ & 28 & 0.6508 & rebound \\
5 & $0^{\circ}$ & 32 & 0.4399 & adhesion \\
6 & $0^{\circ}$ & 32 & 0.4851 & rebound \\
7 & $30^{\circ}$ & 23 & 0.8637 & adhesion \\
8 & $30^{\circ}$ & 23 & 0.8920 & rebound \\
9 & $30^{\circ}$ & 28 & 0.6003 & adhesion \\
10 & $30^{\circ}$ & 28 & 0.6247 & rebound \\
11 & $30^{\circ}$ & 32 & 0.3811 & rebound \\
12 & $30^{\circ}$ & 32 & 0.4052 & adhesion \\
13 & $45^{\circ}$ & 18 & 1.0531 & rebound \\
14 & $45^{\circ}$ & 18 & 1.0864 & adhesion \\
15 & $45^{\circ}$ & 23 & 0.7855 & rebound \\
16 & $45^{\circ}$ & 23 & 0.8037 & adhesion \\
17 & $45^{\circ}$ & 28 & 0.4926 & rebound \\
18 & $45^{\circ}$ & 28 & 0.5110 & \\
\hline
\end{tabular}

The main reason for the difference of the critical capture velocity predicted by the three models is that Hertz model only considers the influence of the repulsion force and energy dissipation in the collision process between two particles, while DMT model and BD model further consider the influence of the attractive force on the collision process based on Hertz model. The DMT model holds that attractive force exists outside the contact region of two particles, while the $\mathrm{BD}$ model holds that attractive force exists within the contact region of two particles. This results in the minimum critical capture velocity predicted by the Hertz model, the middle one predicted by the DMT model, and the maximum one predicted by the BD model.

\subsubsection{Effect of Particle Diameter and Incident Angle on Critical Capture Velocity}

Figure 9 shows the relationship between particle diameter and critical capture velocity predicted by the $\mathrm{BD}$ model at three different incidence angles. At the same incident angle, the critical capture velocity predicted by the BD model decreases with the increase of particle diameter. This indicates that the smaller the particle diameter of coal ash is, the easier it is to be captured on the stainless steel plate surface. There have also been reports that smaller particles are more likely to be captured [6]. In addition, for coal ash particles with the same diameter, the critical capture velocity predicted by the BD model also decreases with the increase of incident angle. This is consistent with the existing studies that particles are more easily captured at small incidence angles $[20,22]$. This means that the tangential component of the incident velocity has an effect on the critical capture velocity of the particle, which may be caused by the particle rotation caused by the tangential component of the incident velocity. Under the same conditions, the normal collision process is easier to capture than the oblique collision process. 


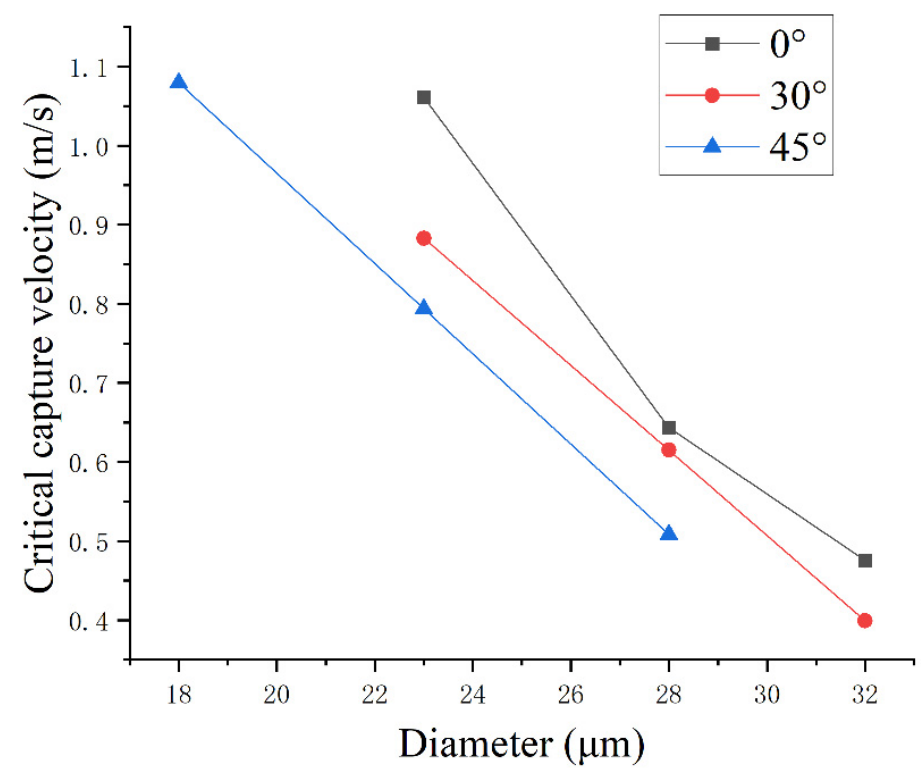

Figure 9. The variation curve of critical capture velocity with particle diameter predicted by BD model under three different incident angles.

\section{Conclusions}

In this paper, the impact process of coal ash particles with the stainless steel surface at different incident angles was filmed by high-speed digital camera technology, and the influence of incident normal velocity on normal restitution coefficient was analyzed and obtained. In addition, based on Hertz theory, DMT theory, and BD theory, the motion equation is established to predict the critical damping coefficient and the critical capture velocity of coal ash particles impacting on the stainless steel surface, and the influence of particle diameter on the critical capture velocity is analyzed. The main conclusions are as follows.

The incident normal velocity has great influence on the normal restitution coefficient. When the incident normal velocity of coal ash particles increases, the normal restitution coefficient first increases and then decreases. The corresponding fitting curve produces an ascending region, a transition region, and a descending region.

The Hertz model, DMT model, and BD model were introduced to predict the movement behavior of coal ash particles impact on the stainless steel surface. The critical damping coefficient and the critical capture velocity are calculated. By comparing with the experimental values, it is found that the critical capture velocity predicted by the BD model is closer to the actual situation. Taking the coal ash particle of $23 \mu \mathrm{m}$ as an example, the critical capture velocity predicted by the $\mathrm{BD}$ model is $1.0611 \mathrm{~m} / \mathrm{s}$ at the incident angle of $0^{\circ}, 0.8829 \mathrm{~m} / \mathrm{s}$ at the incident angle of $30^{\circ}$, and $0.7940 \mathrm{~m} / \mathrm{s}$ at the incident angle of $45^{\circ}$.

The critical capture velocity decreases with the increase of particle diameter. In addition, for particles with the same particle diameter, the critical capture velocity of particles decreases with the increase of incident angle.

Author Contributions: Data curation, J.X. and H.M.; Methodology, H.M., C.L., and S.L.; Writingoriginal draft, J.X.; Writing-review and editing, Z.Z. and Z.F. All authors have read and agreed to the published version of the manuscript.

Funding: This research was funded by National Natural Science Foundation of China (NSFC Grant No. 51906164), Liaoning Province Nature Fund Guidance Plan (No. 2019-ZD-0248), and Liaoning Education Department of 'Seed-ling Project' for Young Scientific and Technological talents-General Youth Training Program Project (No. JYT19021).

Institutional Review Board Statement: Not applicable.

Informed Consent Statement: Not applicable. 
Data Availability Statement: Data sharing is not applicable.

Acknowledgments: The authors would like to acknowledge Dong Ming (Dalian University of Technology) for providing the right to use the experimental apparatus.

Conflicts of Interest: The authors declare no conflict of interest.

\section{Nomenclature}

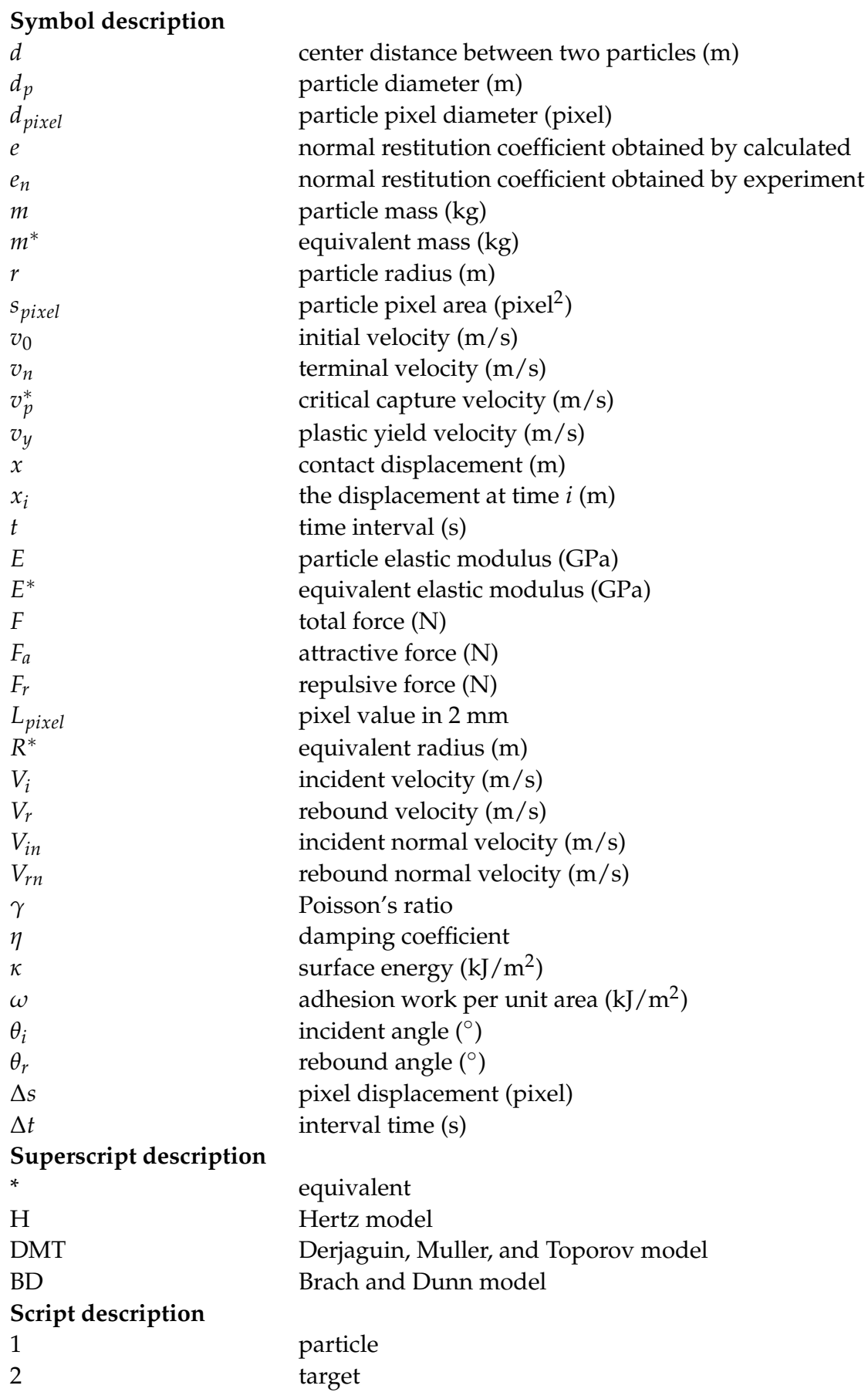

\section{References}

1. Dahneke, B. The capture of aerosol particles by surfaces. J. Colloid Interface Sci. 1971, 37, 342-353. [CrossRef]

2. Dahneke, B. Measurements of bouncing of small latex spheres. J. Colloid Interface Sci. 1973, 45, 584-590. [CrossRef]

3. Dahneke, B. Further measurements of the bouncing of small latex spheres. J. Colloid Interface Sci. 1975, 51, 58-65. [CrossRef] 
4. Rogers, L.N.; Reed, J. The adhesion of particles undergoing an elastic-plastic impact with a surface. J. Phys. D Appl. Phys. 1984, 17, 677. [CrossRef]

5. Dunn, P.F.; Brach, R.M.; Janson, G.G. Surface-Contact Mechanics During Oblique Impact of Microspheres with Planar Surfaces. Aerosol Sci. Technol. 1996, 25, 445-465. [CrossRef]

6. Li, X.; Dunn, P.F.; Brach, R.M. Experimental and numerical studies on the normal impact of microspheres with surfaces. J. Aerosol Sci. 1999, 30, 439-449. [CrossRef]

7. Zhang, Y.; Fang, Z.; Zhao, S.; Wei, M.; Wu, X.; Sun, L. An experimental study on the wall collision of micro-sized graphite particles by high-speed photomicrography. Prog. Nucl. Energy 2020, 125, 103391. [CrossRef]

8. Kim, O.V.; Dunn, P.F. Direct visualization and model validation of microsphere impact and surface capture. J. Aerosol Sci. 2008, 39, 373-375. [CrossRef]

9. Sommerfeld, H.; Koch, C.; Schwarz, A.; Beck, A. High velocity measurements of particle rebound characteristics under erosive conditions of high pressure compressors. Wear 2021, 470, 203626. [CrossRef]

10. Tiamiyu, A.A.; Sun, Y.; Nelson, K.A.; Schuh, C.A. Site-specific study of jetting, bonding, and local deformation during highvelocity metallic microparticle impact. Acta Mater. 2021, 202, 159-169. [CrossRef]

11. Wall, S.; John, W.; Wang, H.-C.; Goren, S.L. Measurements of Kinetic Energy Loss for Particles Impacting Surfaces. Aerosol Sci. Technol. 1990, 12, 926-946. [CrossRef]

12. Ming, D.; Li, S.; Xie, J.; Jian, H. Experimental Studies on the Normal Impact of Fly Ash Particles with Planar Surfaces. Energies 2013, 6, 3245-3262.

13. Li, S.; Xie, J.; Dong, M.; Bai, L. Rebound characteristics for the impact of SiO2 particle onto a flat surface at different temperatures. Powder Technol. 2015, 284, 418-428. [CrossRef]

14. Li, X.; Dong, M.; Li, S.; Shang, Y. Experimental and theoretical studies of the relationship between dry and humid normal restitution coefficients. J. Aerosol Sci. 2019, 129, 16-27. [CrossRef]

15. Konstandopoulos, A.G. Particle sticking/rebound criteria at oblique impact. J. Aerosol Sci. 2006, 37, 292-305. [CrossRef]

16. Aylor, D.E.; Ferrandino, F.J. Rebound of pollen and spores during deposition on cylinders by inertial impaction. Atmos. Environ. 1985, 19, 803-806. [CrossRef]

17. Gibson, L.; Gopalan, B.; Pisupati, S.V.; Shadle, L.J. Image analysis measurements of particle coefficient of restitution for coal gasification applications. Powder Technol. 2013, 247, 30-43. [CrossRef]

18. Kuuluvainen, H.; Arffman, A.J.; Rvinen, A.; Harra, J.; Keskinen, J. The effect of materials and obliquity of the impact on the critical velocity of rebound. Aerosol Sci. Technol. 2017, 51, 301-310. [CrossRef]

19. Sondergaard, R.; Chaney, K.; Brennen, C.E. Measurements of Solid Spheres Bouncing Off Flat Plates. J. Appl. Mech. 1990, 57, 694-699. [CrossRef]

20. Xie, J.; Zhu, Z.; Yang, T.; Dong, M.; Li, R. The effect of incident angle on the rebound behavior of micro-particle impacts. J. Aerosol Sci. 2021, 155. [CrossRef]

21. Brach, R.M.; Dunn, P.F.; Li, X. Experiments and Engineering Models of Microparticle Impact and Deposition. J. Adhes. 2000, 74, 227-282. [CrossRef]

22. Beek, V.M.; Rindt, C.C.; Wijers, J.J.; Steenhoven, V. Rebound characteristics for $50-\mu \mathrm{m}$ particles impacting a powdery deposit. Powder Technol. 2006, 165, 53-64. [CrossRef]

23. Li, X.; Dunn, P.F.; Brach, R.M. Experimental and Numerical Studies of Microsphere Oblique Impact with Planar Surfaces. J. Aerosol Sci. 2000, 31, 583-594. [CrossRef]

24. Maugis, D. Contact, Adhesion and Rupture of Elastic Solids; Springer: Berlin/Heidelberg, Germany, 2000.

25. Derjaguin, B.V.; Muller, V.M.; Toporov, Y.P. Effect of contact deformations on the adhesion of particles. J. Colloid Interface Sci. 1975, 53, 314-326. [CrossRef]

26. Brach, R.M.; Dunn, P.F. Macrodynamics of Microparticles. Aerosol Sci. Technol. 1995, 23, 51-71. [CrossRef]

27. Thornton, C.; Ning, Z. A theoretical model for the stick/bounce behaviour of adhesive, elastic-plastic spheres. Powder Technol. 1998, 99, 154-162. [CrossRef]

28. Matsunaga, T.; Kim, J.K.; Hardcastle, S. Crystallinity and selected properties of fly ash particles. Mater. Sci. Eng. A 2002, 325, 333-343. [CrossRef]

29. Ledbetter, H.M. Stainless-steel elastic constants at low temperatures. J. Appl. Phys. 1981, 52, 1587-1589. [CrossRef] 